\title{
Impacto de la satisfacción laboral en el desempeño de un grupo de policías estatales
}

\author{
Oliverio Leonel Linares Olivas ${ }^{1}$ Cosme Francisco Maldonado Rivera ${ }^{2}$ \& Rodolfo Enrique Gutiérrez \\ Martínez ${ }^{3}$
}

Recibido:26/02/2018 Aceptado: 09/09/2018

DOI: $10.21772 /$ ripo.v37n1a01

\begin{abstract}
Resumen
El objetivo del estudio fue evaluar el impacto de algunas facetas de la satisfacción laboral sobre el desempeño de un grupo de policías. Participaron 242 personas de una corporación de seguridad pública. Se realizó análisis discriminante tomando en consideración los aspectos teóricos que están relacionados con el desempeño: satisfacción con el trabajo mismo, relaciones con compañeros, relaciones con el jefe y salario y prestaciones. Una tercera parte de los policías reportaron baja satisfacción laboral general. Los niveles más bajos se reportaron en relación a la naturaleza misma del trabajo. Los cuatro factores de la satisfacción laboral se relacionaron de manera estadísticamente significativa con el desempeño. El aspecto que cobró mayor relevancia fueron las relaciones con los compañeros de trabajo. Las relaciones con los compañeros cobran una importancia fundamental no sólo en el desempeño laboral, sino también en atenuar los riesgos que tal labor implica.
\end{abstract}

Palabras clave: psicología policial, policía, desempeño laboral, satisfacción laboral, desarrollo organizacional.

\section{Impact of job satisfaction in work performance in a group of state police}

\begin{abstract}
The aim of this paper was to assess the impact of some facets of job satisfaction on the performance of a group of 242 police officers. Discriminant analysis was used taking into consideration theoretical aspects related with job performance: job satisfaction in general, relationships with coworkers, relationships with superiors and salary and benefits. One third of the cops was found with low generalized job satisfaction, where lowest levels were in relationship with the nature of the job. The four factors of job satisfaction had statistical significance in relationship with performance. The aspect with higher relevance was coworker's relationships. They take fundamental importance not only in job performance, but also in moderate risks associated with the job.
\end{abstract}

Keywords: Police psychology, police, work performance, job satisfaction, organizational development. 1 Doctor en psicología social y ambiental, Universidad Nacional Autónoma de México. Universidad Juárez del Estado de Durango. Facultad de Psi-
cología y Terapia de la Comunicación Humana. E-mail: leonel@ujed.mx

2 Magister. Universidad Juárez del Estado de Durango. Facultad de Psicología y Terapia de la Comunicación Humana. E-mail: cfmaldonador@ujed.mx

3 Doctor en sociología, Universidad de París. Universidad Nacional Autónoma de México, Facultad de Psicología. E-mail: rodolfo@unam.mx

Cómo citar este artículo: Linares Olivas, O. L.; Maldonado Rivera, C. F. \& Gutiérrez Martínez, R. E. (2018). Impacto de la satisfacción laboral en el desempeño de un grupo de policías estatales. Revista Interamericana de Psicología Ocupacional, 37(1), 6-16 DOI: 10.21772/ripo.v37n1a01 


\section{Introducción}

En la dinámica de la organización social es inherente el derecho a la seguridad, la sensación en la ciudadanía de "sentirse seguro" es decir, la tranquilidad pública, como apuntaría Rousseau. El control, el castigo y la prevención del delito son aspectos fundamentales en el orden social de cualquier nación. Sin duda, la inseguridad pública que afecta a México es uno de los problemas sociales que ha cobrado mayor relevancia en los últimos años, no sólo en los aspectos políticos y sociales, sino también en el riesgo para aquellos que se dedican a esta actividad. Es por ello que desde diversas ópticas se han abordado las repercusiones de esta situación. Una de estas miradas, y quizá la más estudiada, es la del impacto de las fuerzas armadas en el estrés de la población civil. Por ejemplo, Leiner y Jiménez (2011), destacan la importancia que ha tenido la inseguridad en estudiantes universitarios, donde observaron que la violencia a nivel local incrementa la percepción de estrés en la población. Otro enfoque de análisis (Linares, 2015) se ha centrado en los síntomas de estrés postraumático de la población civil a causa de la guerra contra el narcotráfico. Allí se encontró que el $56 \%$ de las personas que habían presenciado, atestiguado o directamente haber sido víctimas de la violencia presentaban síntomas entre moderados y graves de este trastorno.

Desde las instituciones policíacas, resulta prioritario mirar también hacia la experiencia de quienes desempeñan la actividad policial. Uno de los tópicos más significativos es el que se refiere a las condiciones bajo las cuales los policías hacen frente a la delincuencia y en cómo su actividad repercute en la sociedad (Ménard \& Arter, 2013).

Como describen Milla, Sanz, Apellaniz y Pascual (2001), el policía se desempeña en un medio hostil de marginalidad y criminalidad, e inclusive, sus propias herramientas de trabajo (armas) son también agentes estresores para ellos mismos. Al mismo tiempo que este tipo de trabajadores está expuesto a los agentes estresores propios de su actividad, hay factores estructurales característicos de la institución que son elementos generadores de tensión (Marmar et al., 2006). Así, el peso de los aspectos institucionales sobre la satisfacción en el personal de policía es evidente. Por ello, resulta determinante la experiencia desde la cual el policía realiza su labor, entre otros aspectos, el grado de satisfacción que percibe de su trabajo. La satisfacción laboral se refiere a la evaluación que una persona realiza de diversos factores de su trabajo, de la comparación resultante entre el esfuerzo que imprime a sus labores y la recompensa recibida. Aunado a lo anterior, lo que espera o desea obtener del trabajo en comparación con lo que otros reciben y lo que efectivamente recibe (Adams \& Freedman, 1976).

Abdulla, Djebarni y Mellahi (2011) encontraron que la estrategia y las políticas organizacionales tienen su principal impacto en la satisfacción laboral, seguido de los ingresos y las prestaciones, las relaciones con los compañeros y el jefe. Además, las variables demográficas relacionadas con satisfacción fueron: edad, tener pareja sentimental, ocupar puestos de mando y años de experiencia. Tuckey, Chrisopoulos y Dollard (2012), postulan que las condiciones organizacionales como las relaciones con el jefe, con los compañeros de trabajo, el ingreso económico y el gusto con el que se realiza el trabajo son factores relacionados directamente con el desempeño laboral. En particular, se tiene evidencia de que algunos de los mejores predictores de la satisfacción con el trabajo se refieren a la naturaleza del trabajo mismo y a los compañeros (Chiang \& Ojeda, 2013; Dehue, Bolman, Völlink, \& Pouwelse, 2012; Linares \& Gutiérrez, 2010).

La satisfacción laboral se ha correlacionado con el desempeño en el trabajo (Buitendach \& De Witte, 2005; Christen, Lyer, \& Soberman, 2006; Hunt, Chonko, \& Wood, 1985; Ostroff, 1992). Particularmente, Marín y Placencia (2017) encontraron una relación positiva entre el nivel 
satisfacción y motivación, sobre todo en las relaciones con el jefe y con los compañeros de trabajo. En grupos de empleados de la función pública, Chiang y San Martín (2014) hallaron una correlación estadísticamente significativa entre los niveles de desempeño, la productividad y la satisfacción laboral con el jefe.

En el ámbito policial se ha encontrado que la confusión del rol y la influencia del líder pueden ser predictores de conflictos entre los compañeros de trabajo, lo cual afecta negativamente el clima organizacional y el desempeño (Hauge et al., 2011). Además, Liu, Liu, Mills y Fan (2013) indican que existen estresores del rol como conflictos interpersonales, obstáculos organizacionales y el peligro inherente de la labor policíaca que perjudican el rendimiento.

Como una medida de afrontamiento, estos autores refieren que aquellos policías que tienen más dedicación o compromiso con la labor policíaca se desempeñan mejor a pesar de los estresores mencionados, incluso la necesidad de trabajar en turnos nocturnos, aunado al riesgo y fatiga que eso implica. Evidentemente, las jornadas de 24 por 48 horas son necesarias en las instituciones de seguridad y en equipos de primer contacto en emergencias (policía, soldados, bomberos) o en momentos de atención a desastres naturales o crisis sociales. A este respecto, hay diversas investigaciones que han enfatizado las consecuencias negativas en la salud y la satisfacción en general debido a rotar turnos por la alteración de los ciclos circadianos. Por ejemplo, Feo (2008) recopiló los resultados de 23 estudios que analizaron la rotación de horarios y afectación a la salud. Principalmente, encontró que los trastornos músculo-esqueléticos y los trastornos del sueño encabezaban la lista de afecciones físicas y, en la esfera social, las relaciones familiares y con los amigos fueron las más perjudicadas.

Además de la rotación de turnos, otro de los factores que tiene repercusiones en la salud y el desempeño, son las relaciones con el jefe (Boudrias et al., 2011). El grado en que un empleado percibe una buena o mala relación con el supervisor es de suma importancia, pues, es éste el que se encarga de las instrucciones de trabajo y de la evaluación de su desempeño, lo cual conlleva repercusiones con su compromiso laboral (O'Shea, Goodwin, Driskell, Salas \& Ardison, 2009). Es sabido que en un ambiente como en el que nos ocupa esta investigación, no hay una concepción de disposición para discutir las instrucciones con los mandos, ya que (al igual que en ambientes militares) la desobediencia a un superior puede ser castigada de forma enérgica.

En este sentido, Sánchez, Bray, Vincus y Bann (2004) encontraron que algunos de los principales predictores de insatisfacción laboral en este tipo de oficios era la percepción de presión en el trabajo debida a los mandos y a los compañeros de trabajo. Aunque los autores también reconocen que este tipo de estresores son característicos y forman parte de la propia actividad que se desempeña. Es por eso que la propia actividad de policía es estresante en sí misma. Así, resulta muy difícil, si no imposible, eliminarlos. Más bien se opta por la mejor eficiencia de las estrategias de afrontamiento para tales estresores.

Ahora bien, la medición del desempeño de los policías se ha enfocado tradicionalmente, a valoraciones econométricas a través del número de elementos efectivos, disponibilidad de armas, índice de temor y de la valoración que la sociedad realiza de los cuerpos policíacos (Biggam, Power, Mcdonald, Carcary, \& Moodie, 1997; Tudela, 2012). Sin embargo, otra forma de evaluar el desempeño es a través de la manera en la que el trabajador percibe que hace sus propias labores (Reese, 1986). La propia evaluación y seguridad del desempeño cobran gran relevancia en actividades de riesgo, ya que la integridad e incluso la vida de otras personas dependen de sus decisiones, acciones, omisiones e inclusive, las dudas que se presentan en situaciones de emergencia (Gutiérrez \& Ángeles, 2012). La 
propia reflexión del desempeño realizado es una oportunidad para conocer el grado en el cual se han alcanzado las metas de trabajo, los planes de acción a seguir, así como determinar el grado en que hay efectividad y precisión en el trabajo (Gutiérrez \& Ángeles, 2012; Ivancevich \& Matteson, 1991).

La relación entre la satisfacción laboral y el desempeño puede ser analizada desde dos direcciones. Por un lado, es la satisfacción la que promueve un mejor desempeño o bien, es la ejecución del buen desempeño un aliciente para sentir que el trabajo se ha hecho bien $y$ esto generar satisfacción. De hecho, algunos estudios demuestran correlación significativa entre el nivel de satisfacción y el desempeño (Atalaya, 1999; Colín \& Simón, 2014). Por lo tanto y considerando lo expuesto, en la presente investigación se buscó identificar aquellos factores de la satisfacción laboral que inciden en la valoración del desempeño laboral en un grupo de policías municipales. Tomando como punto de partida que hay evidencia de que los factores de la satisfacción laboral inciden en el desempeño. Sobre todo, tratándose de personal que trabaja bajo un contexto de riesgo constante y en el que se encuentra comprometida su seguridad así como la de la población.

\section{Método}

\section{Participantes}

Se encuestó a 242 policías del estado de Durango durante las horas de trabajo mediante un muestreo por conveniencia. La mayoría hombres (78\%), y el promedio de edad fue de 38 años, con un rango entre 22 y 69 . El 76\%, reportó tener pareja sentimental y respecto al nivel de estudios, el $70 \%$ contaba con instrucción de secundaria y bachillerato. La mayor parte $(80 \%)$ laboraba bajo un modelo de trabajo mixto, es decir, rotando turnos. La selección de la muestra fue por conveniencia debido a las características del trabajo (como la rotación de turnos) que hicieron inviable un muestreo probabilístico. La aplicación se realizó en el mes de mayo de 2016.

\section{Instrumentos}

Para evaluar la satisfacción laboral se utilizó la escala de satisfacción laboral (ESL) de García (1985), creada para la población mexicana. Se define a la Satisfacción laboral como la emoción placentera o estado emocional agradable que es resultado de la apreciación positiva del trabajo y/o de las experiencias laborales (Locke, 1976, en Gutiérrez \& Ángeles, 2012). La ESL es un instrumento de lápiz y papel que consta de 22 reactivos estilo Likert que evalúa cuatro factores de la satisfacción: con el trabajo mismo, compañeros de trabajo, ingreso y prestaciones, y relaciones con el jefe. El alfa de Cronbach obtenido para la escala total fue de .95 (García, 1985).

Para la autoevaluación del desempeño se utilizó la sub escala de Desempeño del SWS - Survey (C) de Gutiérrez y Ostermann (1994) que consta de 13 indicadores: cantidad, calidad, moral/actitud, disciplina, trabajo en equipo, responsabilidad, asistencia, iniciativa, organización, eficiencia, ritmo, cooperación y compromiso. Es de lápiz y papel, donde se auto evalúa el desempeño en un rango que va desde pobre (1) a excelente (7).

\section{Procedimiento}

Previa autorización de las autoridades policíacas se procedió a la aplicación de los instrumentos a lo largo de tres días de forma grupal. Al finalizar se completó el $30 \%$ del estado de fuerza de ese momento. A todos los participantes les fue leída una carta de consentimiento informado notificando la voluntariedad, anonimato y confidencialidad de los resultados. Los cálculos se realizaron con 
los reactivos efectivamente contestados y se desecharon los formatos incompletos.

Se efectuaron análisis de estadística descriptiva para delinear los datos demográficos, los porcentajes de satisfacción general (cuatro factores) y para puntuar las 13 autovaloraciones de la escala de Desempeño. Posteriormente, se realizaron comprobaciones de curtosis y simetría para evaluar la distribución normal de las variables. Después, se utilizó análisis de correlación de Spearman para analizar los cuatro factores y correlación producto momento de Pearson. Para determinar la influencia entre de cada una de las variables de satisfacción (factores) sobre el desempeño total se utilizó la técnica de análisis discriminante (Tabachnick \& Fidell, 1989). Se usó el programa estadístico IBM SPSS versión 20.

\section{Resultados}

Los resultados obtenidos en la escala de satisfacción laboral se muestran de manera global incluyendo sus subescalas en la Tabla 1.

Tabla 1. Descriptivos escala de satisfacción laboral

\begin{tabular}{lcc}
\hline Variables & $M$ & $D E$ \\
\hline Satisfacción laboral (Total) & 70.77 & 14.13 \\
Satisfacción con el trabajo mismo & 19.09 & 3.05 \\
Satisfacción con el jefe & 15.81 & 5.60 \\
Satisfacción con el salario y & 17.13 & 5.29 \\
prestaciones & 14.56 & 3.42 \\
Satisfacción con los compañeros &
\end{tabular}

Con respecto a la escala de desempeño se observó una puntuación media de 79.16 con una desviación estándar de 11.267. Sin embargo, al llevar a cabo la prueba de normalidad de Kolmogorov-Smirnoff el resultado de la misma fue negativo $(p<.001)$ por lo que las pruebas paramétricas para esta variable no parecieron ser una opción viable. La distribución de los resultados puede ser constatada en la gráfica siguiente:

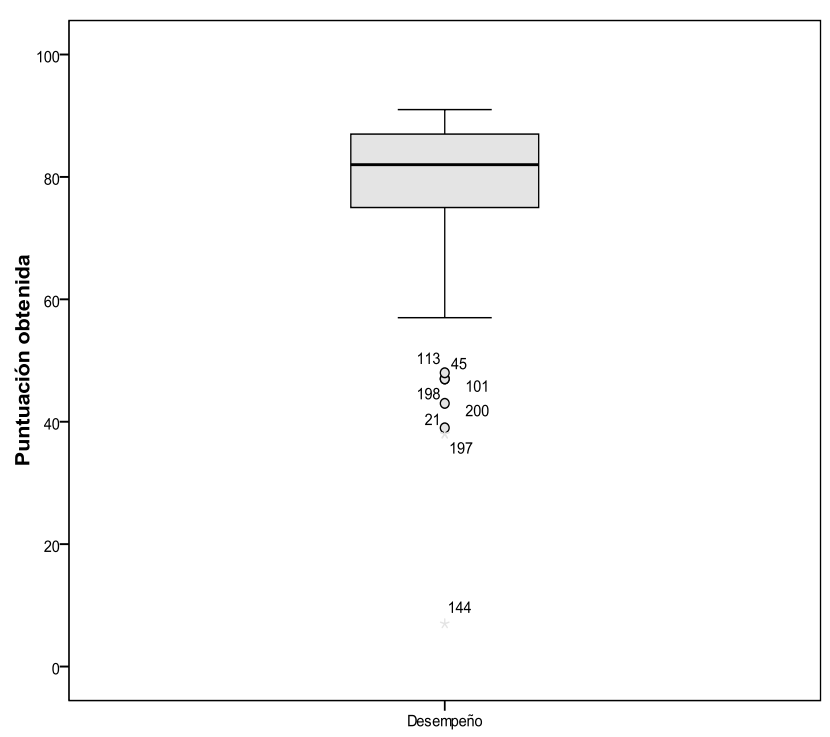

Figura 1. Distribución de las puntuaciones obtenidas en la escala de desempeño

Como puede observarse por inspección visual, los resultados están sesgados hacia la parte alta de la escala. Los cuartiles de esta escala, los cuales se emplearon para hacer los análisis subsecuentes, se reportan en la tabla a continuación:

Tabla 2. Cuartiles de la distribución de puntuaciones de la escala de desempeño

\begin{tabular}{cc}
\hline Cuartil & Puntuación \\
\hline $\mathrm{Q}_{0}$ & 7 \\
$\mathrm{Q}_{1}$ & 75 \\
$\mathrm{Q}_{2}$ & 82 \\
$\mathrm{Q}_{3}$ & 87 \\
$\mathrm{Q}_{4}$ & 91 \\
\hline
\end{tabular}

Con el propósito de poder observar una tendencia en la relación entre las variables, se procedió a visualizar la distribución de las puntuaciones de satisfacción laboral clasificando los resultados de acuerdo con el cuartil obtenido en la escala de desempeño. Los resultados de la escala global y de las escalas pueden ser apreciados en las figuras que son precedidas por este párrafo. 


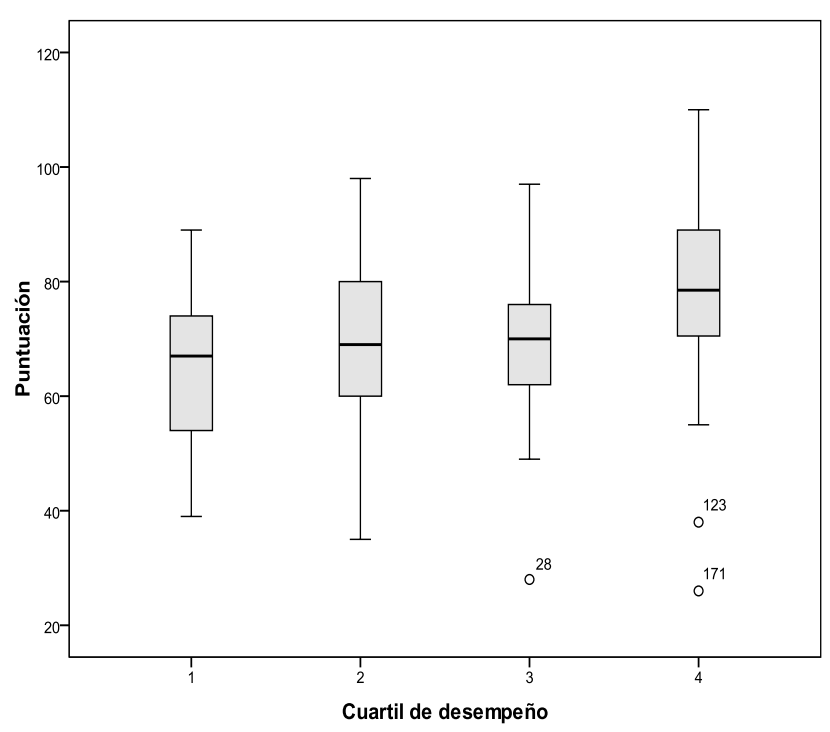

Figura 2. Distribución de las puntuaciones de satisfacción laboral clasificadas de acuerdo con el cuartil de la puntuación obtenida en la escala de la desempeño

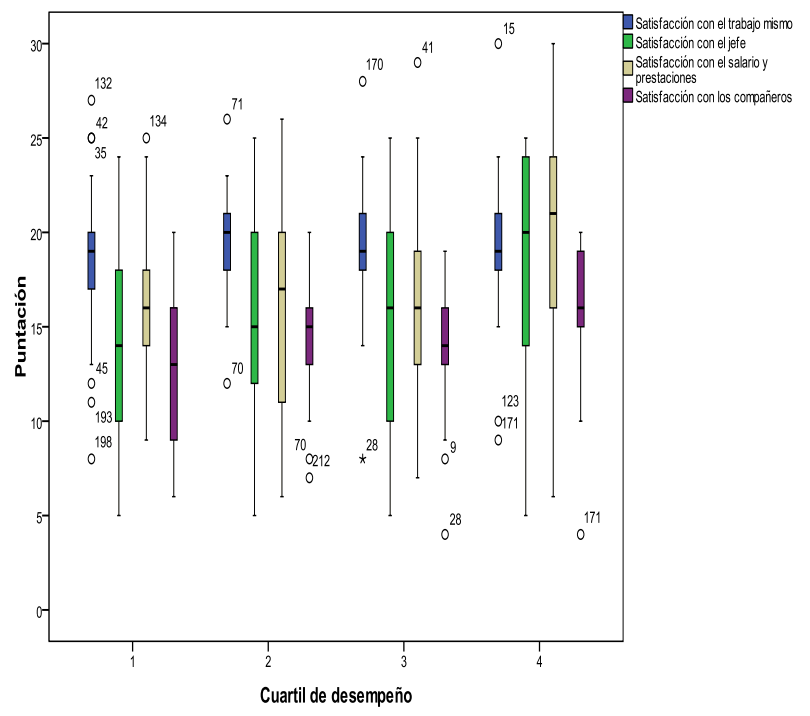

Figura 3. Puntuaciones de las subescalas de satisfacción laboral clasificadas de acuerdo al cuartil de la puntuación obtenida en la escala de desempeño
La inspección visual de las gráficas permite observar que los valores de satisfacción laboral obtenidos en los tres primeros cuartiles de la escala de desempeño son similares, mientras se observa una elevación de las puntuaciones en el cuartil cuatro, el del desempeño más alto. Tomando esto en consideración se decidió clasificar los resultados de este último constructo en dos categorías, alto desempeño para las puntuaciones del cuartil $4 \mathrm{y}$ no alto desempeño para las restantes.

Un primer análisis mediante la prueba $\mathrm{t}$ de Student mostró diferencias significativas $(p<.001)$ entre las medias de las puntuaciones obtenidas entre los grupos de policías que consideran alto desempeño y no alto desempeño. Además, se encontró un incremento de 10.34 puntos en el grupo que se describe a sí mismo como pertenecientes a la categoría de alto desempeño ().

Tabla 3. Estadísticos descriptivos de Satisfacción Laboral según grupos desempeño

\begin{tabular}{lcccc}
\hline $\begin{array}{l}\text { Alto } \\
\text { desempeño }\end{array}$ & $N$ & $M$ & $D E$ & $S E$ \\
\hline No & 139 & 67.82 & 12.90 & 1.09 \\
$\mathrm{Si}$ & 56 & 78.16 & 14.54 & 1.94 \\
\hline
\end{tabular}

En una siguiente fase del análisis $y$ con la finalidad de responder a la pregunta de investigación se procedió a probar un modelo predictivo. Se optó por el análisis discriminante, que de acuerdo con Tabachnik y Fidell (1989), así como a Hair et al. (2010) es una técnica apropiada para este caso dado que se tiene una variable categórica (alto desempeño) que puede ser predicha mediante una función de variables de intervalo o proporcionales (componentes de la satisfacción laboral) cuyos valores clasifiquen (discriminen) el resultado de la categórica (ver Tabla 4). 
Tabla 4. Prueba Lambda de Wilks para diferencia de medias en los predictores en el análisis discriminante del alto desempeño mediante las subescalas de satisfacción laboral

\begin{tabular}{lccccc}
\hline Variable & $\begin{array}{c}\text { Lambda } \\
\text { de Wilks }\end{array}$ & $F$ & $d f 1$ & $d f 2$ & $p$ \\
\hline $\begin{array}{l}\text { Satisfacción con } \\
\text { el trabajo mismo }\end{array}$ & .997 & .493 & 1 & 194 & .483 \\
$\begin{array}{l}\text { Jefe } \\
\begin{array}{l}\text { Salario y } \\
\text { prestaciones }\end{array}\end{array}$ & .903 & 20.845 & 1 & 194 & $<.001$ \\
Compañeros & .921 & 16.689 & 1 & 194 & $<.001$ \\
\hline
\end{tabular}

Los resultados de la prueba Lambda de Wilks arrojaron que tres de las subescalas de satisfacción laboral muestran que las medias de los grupos de alto desempeño afirmativo $\mathrm{y}$ negativo son estadísticamente diferentes entre sí. Sólo el factor de satisfacción con el trabajo mismo no mostró tener una relación que permita discriminar entre grupos.

Con respecto a la calidad de los datos como predictores la prueba $\mathrm{M}$ de Box de homogeneidad de varianzas arrojó una $p=0.77$. Adicionalmente, la correlación canónica reportada en el modelo fue de 0.357 que es moderada. Sin embargo el valor de chi cuadrado de la prueba Lambda de Wilks del modelo es $<.001$ que muestra centroides claramente diferenciados en las categorías clasificadas mediante la función discriminante. Esto indica viabilidad en el análisis y, por lo tanto, cumplen los requisitos para proseguir con el mismo.

La función discriminante estandarizada resultado del análisis fue:

$$
\begin{gathered}
z \mathrm{D}=.502 z(\text { salario } y \\
\text { prestaciones })+.694 z(\text { compañeros })
\end{gathered}
$$

Esto demuestra que se puede predecir la pertenencia a los grupos de alto desempeño afirmativo y negativo considerando sólo los resultados de dos de las subescalas, siendo el mejor predictor la satisfacción con los compañeros. La capacidad predictiva del modelo se puede estimar mediante la prueba de clasificación que arrojó un $67.1 \%$ de los casos ubicados adecuadamente por este modelo. Por otro lado, la matriz de estructura permite evaluar la correlación entre los predictores y la función discriminante.

Tabla 5. Matriz de estructura del modelo de función discriminante

\begin{tabular}{lc}
\hline Variable & Correlación con el modelo \\
\hline Compañeros & .886 \\
Salario y prestaciones & .768 \\
Jefe & .686 \\
$\begin{array}{l}\text { Satisfacción con el trabajo } \\
\text { mismo }\end{array}$ & .440 \\
\hline
\end{tabular}

Esta tabla permite ver que la variable jefe, aunque no fue incluida en el modelo, sí guarda una correlación a considerar en relación con el desempeño.

\section{Discusión y conclusiones}

El objetivo de este estudio fue analizar los efectos de la satisfacción laboral en el desempeño de un grupo de policías. Se observó que 35\% reportó baja satisfacción laboral general, que ésta es menor en las mujeres policías, y además, que es independiente del nivel de estudios. Como primera reflexión, es de destacar el bajo nivel de satisfacción con la naturaleza misma del trabajo (41\%) pues éste es uno de los elementos preponderantes para desempeñar bien un oficio o actividad. Sin embargo, hay que considerar que el oficio de policía ha sido catalogado como uno de los más peligrosos en el mundo. En 2013 el diario "The Sun” enlistó los trabajos más riesgosos en el orbe. El de la policía de México se posicionó en décimo lugar, detrás de los periodistas en Siria, mineros de carbón en China y agentes de seguridad privada en Irak. Considerando esto, no es difícil notar que desafortunadamente pocas personas desean ser parte de la policía mexicana, máxime, cuando la sociedad es testigo de los notables eventos de corrupción en los que se encuentran 
implicados muchos elementos policíacos. Ello, a pesar de las evaluaciones de los centros de control de confianza.

Un hallazgo importante fue el papel de la naturaleza social el trabajo de policía, ya que los resultados mostraron que el nivel de satisfacción laboral en el factor de las relaciones con los compañeros es determinante para discriminar un alto y bajo desempeño.

La relación que tiene el policía con su compañero de trabajo resulta ser el factor principal del desempeño. Como se discutió anteriormente en la sección introductoria, la seguridad e incluso la vida dependen de la dinámica existente entre los compañeros de este tipo de trabajo. Esto coincide en otras actividades laborales, donde se ha observado que la buena relación con compañeros es muy relevante en la satisfacción laboral y el desempeño (Buitendach \& De Witte, 2005; Chiang y San Martín, 2014; Christen, Lyer, \& Soberman, 2006; Hunt, Chonko, \& Wood, 1985; Linares, 2014; Marín \& Placencia, 2017; Ostroff, 1992). Así, en policías resulta preponderante esta variable por la naturaleza misma de la actividad. Con lo anterior puede suponerse que el acompañamiento y apoyo social que se percibe de parte del compañero de patrulla en las situaciones de riesgo, incertidumbre y peligro parecen ser el elemento más cercano hacia una mejor autopercepción de su desempeño. Por ende, este es el elemento que las autoridades y mandos pueden considerar al momento de diseñar estrategias enfocadas a la mejora del desempeño en cuerpos de seguridad. Adicionalmente, las categorías de satisfacción laboral referidas a ingresos y prestaciones y relaciones con el jefe fueron predictores secundarios de desempeño alto. Esto significa que evidentemente, el reconocimiento monetario así como la posibilidad de obtener mejores condiciones laborales son factores que deben ser tomados en cuenta para mejorar la satisfacción laboral. Sobre todo en un contexto institucional que podría ser catalogado como de muy limitado en el sentido de que los policías ganan apenas unos $\$ 500$ dólares estadounidenses al mes y donde no cuentan con las prestaciones básicas (Secretaría de Gobernación, 2017). Incluso, tienen que costear parte de su uniforme $\mathrm{y}$ herramientas de trabajo. En un contexto de sobrecarga laboral, ya que el estándar mínimo internacional indica que deberían existir 1.8 policías por cada 1000 habitantes, y en México es apenas de 0.8 (Secretaría de Gobernación, 2017).

Un reto particular que enfrentan las autoridades y mandos de la institución se enfoca a la percepción del desempeño. Si bien, los niveles de desempeño percibido son muy elevados en el grupo, resulta prioritario considerar que las categorías puntuadas con menor valor (seis) son aquellas asociadas a una motivación intrínseca, como es la Calidad y la Iniciativa. Así, resulta congruente una menor motivación intrínseca si es que hay, como vimos, baja satisfacción con la naturaleza misma de la actividad laboral (Sprung, \& Jex, 2012). Además, es interesante que la satisfacción con la naturaleza del trabajo fuera el único factor que no se relacionara con alto desempeño, pues ello contradice la visión de que una persona satisfecha con su labor es más productiva, por lo tanto se requerirá seguir esa línea de investigación para abonar más evidencias en ese sentido. Es de reconocer que existen limitaciones en la evaluación en este tipo de empleados debido al difícil acceso para la aplicación de pruebas psicométricas, por lo que es necesario agradecer a las autoridades que facilitaron el desarrollo de esta investigación, así como a los becarios que participaron en la aplicación de los instrumentos.

\section{Referencias}

Abdulla, J., Djebarni, R., \& Mellahi, K. (2011). Determinants of job satisfaction in the UAE: A case study of the Dubai police. Personnel Review, 40(1), 126-146. 
Adams, S. \& Freedman, S. (1976). Equity theory revisited. En Borowitz, L. \& Waldster, E. (Eds.). Advances in experimental social psychology, 9, 43-90. New York: Academic Press.

Atalaya, M. (1999). Satisfacción laboral y productividad. Revista de Psicología, 3(5), Septiembre. Recuperado de: http://sisbib.unmsm.edu.pe/bvrevistas/ psicologia/1999_n5/satisfaccion.htm

Biggam, F. H., Power, K.G., Mcdonald, R.R., Carcary, W.B. \& Moodie, E. (1997). Selfperceived occupational stress and distress in a Scottish police force. Work \& Stress, 11(2), 118-133.

Boudrias, J. S., Desrumaux, P., Gaudreau, P., Nelson, K., Brunet, L., \& Savoie, A. (2011). Modeling the experience of psychological health at work: The role of personal resources, social-organizational resources, and job demands. International Journal of Stress Management, 18(4), 372-395.

Buitendach, J.H., \& De Witte, H. (2005). Job insecurity, extrinsic and intrinsic job satisfaction and affective organizational commitment of maintenance workers in a parastatal. South African Journal of Business Management, 36(2), 27-37.

Chiang, V. \& Ojeda J. (2013). Estudio de la relación entre satisfacción laboral y el desempeño de los trabajadores de las ferias libres. Contaduría y administración, 58(2), 39-60.

Chiang, V. \& San Martín, N. (2014). Análisis de la Satisfacción y el Desempeño Laboral en los Funcionarios de la Municipalidad de Talcahuano. Ciencia \& Trabajo, 17 (54), 159-165 Recuperado de: https://scielo. conicyt.cl/pdf/cyt/v17n54/art01.pdf
Christen, M., Lyer, G., \& Soberman, D. (2006). Job satisfaction, job performance, and Effort: A reexamination using agency theory. Journal of Marketing, 70(1), 137150.

Colín, C. \& Simón, N. (2014). Adicción al trabajo, satisfacción y desempeño laboral en ejecutivos mexicanos. Psicología Iberoamericana. 22(2), 16-24. Recuperado de: http://www.redalyc.org/ $\mathrm{html} / 1339 / 133938134003 /$

Dehue, F., Bolman, C., Völlink, T., \& Pouwelse, M. (2012). Coping with bullying at work and health related problems. International Journal of Stress Management, 19(3), 175-197.

Feo, J. (2008). Influencia del trabajo por turnos en la salud y la vida cotidiana. Tesis de licenciatura. Pontificia Universidad Javeriana, Bogotá, Colombia.

García, B. (1985). Factores relacionados con la satisfacción laboral en mujeres: un estudio comparativo. Tesis de maestría. Facultad de Psicología, Universidad Nacional Autónoma de México.

Gutiérrez, R. \& Ángeles, Y. (2012). Estrés organizacional. Trillas, México.

Gutiérrez, R. \& Ostermann, R. (1994). El SWS -SURVEYC de Salud mental, estrés y Trabajo. Universidad Nacional Autónoma de México, Fairleigh Dickinson University (EUA).

Hair, J., Anderson, R., Tatham, R., y Black, W. (2010). Análisis multivariante. (Esme Prentice y Diego Cano, Trads.). Madrid: Prentice-Hall.

Hauge, L., Einarsen, S., Knardahl, S., Lau, B., Notelaers, G., \& Skogstad, A. (2011). Leadership and role stressors as departmental level predictors of workplace bullying. International Journal of Stress 
Management, 18(4), 305-323.

Hunt, S. D., Chonko, L.B. \& Wood, V.R. (1985). Organizational commitment and marketing. Journal of Marketing, 49(1), 112-127.

Ivancevich, J. \& Matteson, M. (1991). Estrés y trabajo: una perspectiva gerencial. México, Trillas.

Leiner de la Cabada, M. \& Jiménez, P. (2011). Un estudio comparativo del estrés percibido en estudiantes de ciencias administrativas y biológicas en tiempos de violencia. Contaduría y administración. (233), $105-$ 125.

Linares, O. (2014). Influencia del estrés, ansiedad $y$ apoyo en el trabajo sobre el nivel de satisfacción laboral, malestar físico $y$ ausentismo en profesores. Disertación doctoral. Universidad Nacional Autónoma de México. Doi:10.13140/2.1.3581.6004

Linares. O. (Marzo, 2015). Efectos psicológicos de la guerra contra el narcotráfico en habitantes de Durango. Ponencia presentada en el XI Congreso internacional de Salud Mental. Centro Universitario de Ciencias de la Salud, Universidad de Guadalajara. Recuperado de de: https://www.researchgate.net/ publication/273259942_Efectos psicologicos_de_la_guerra_contra_el_ narcotrafico_en_habitantes_de_Durango_ un_estudio_exploratorio

Linares, O., \& Gutiérrez, R. (2010). Satisfacción laboral y percepción de salud mental en profesores. Revista Mexicana de Investigación en Psicología, 2(1), 31-36.

Liu, C., Liu, Y., Mills, M. J., \& Fan, J. (2013). Job stressors, job performance, job dedication, and the moderating effect of conscientiousness: A mixed-method approach. International Journal of Stress
Management, 20(4), 336-363.

Marín, H. \& Placencia, M. (2017). Motivación y satisfacción laboral del personal de una organización de salud del sector privado. Horizonte Médico, 17(4), 42-52. Doi:10.24265/horizmed.2017.v17n4.08

Marmar, C. R., McCaslin, S. E., Metzler, T. J., Best, S., Weiss, D. S., Fagan, J. \& Neylan, T. (2006). Predictors of posttraumatic stress in police and other first responders. Annals of the New York Academy of Sciences, 1071(1), 1-18.

Ménard, K. S., \& Arter, M. L. (2013). Police officer alcohol use and trauma symptoms: Associations with critical incidents, coping, and social stressors. International Journal of Stress Management, 20(1), 3756.

Milla, S., Sanz, B., Apellaniz, G. \& Pascual, I. (2001). Policía y estrés laboral. Estresores organizativos como causa de morbilidad psiquiátrica. Revista de la Sociedad Española de Salud Laboral en la Administración Pública. 1(4), 21 - 25

O'Shea, P. G., Goodwin, G. F., Driskell, J. E., Salas, E., \& Ardison, S. (2009). The many faces of commitment: Facet-level links to performance in military contexts. Military Psychology, 21(1), 5.

Ostroff, C. (1992). The relationship between satisfaction, attitudes, and performance: an organizational level analysis. Journal of Applied Psychology, 77, 963- 974.

Reese, J.T. (1986). Policing the violent society: the American experience. Stress Medicine, 2, 233-240.

Ryan, J. (30 de julio de 2013) Top ten - the world's most dangerous jobs in 2013. The Sun. Recuperado de https://www.thesun. co.uk/archives/news/909276/top-ten-theworlds-most-dangerous-jobs-in-2013/ 
Sánchez, R. P., Bray, R. M., Vincus, A. A., \& Bann, C. M. (2004). Predictors of Job Satisfaction Among Active Duty and Reserve/Guard Personnel in the US Military. Military Psychology 16(1), 19-35.

Secretaría de Gobernación (2017). Diagnóstico nacional sobre las policías preventivas de las entidades federativas. Recuperado de: http://secretariadoejecutivo.gob.mx/doc/ Diagnostico_Nacional_MOFP.pdf

Sprung, J. M., \& Jex, S. M. (2012). Work locus of control as a moderator of the relationship between work stressors and counterproductive work behavior. International Journal of Stress Management, 19(4), 272-291.
Tabachnick, B. \& Fidell, L. (1989). Using multivariate statistics. Boston, MA: Allyn and Bacon.

Tuckey, M. R., Chrisopoulos, S., \& Dollard, M. F. (2012). Job demands, resource deficiencies, and workplace harassment: Evidence for micro-level effects. International Journal of Stress Management, 19(4), 292-310.

Tudela, P. (2012). La evaluación del desempeño de la policía: explorando relaciones entre opinión pública y labor policial en Chile. Revista Criminalidad, 54(1), 341- 366. 